\title{
Cataclysmic Variables and Related Objects
}

A. Evans and Janet H.Wood (eds.)

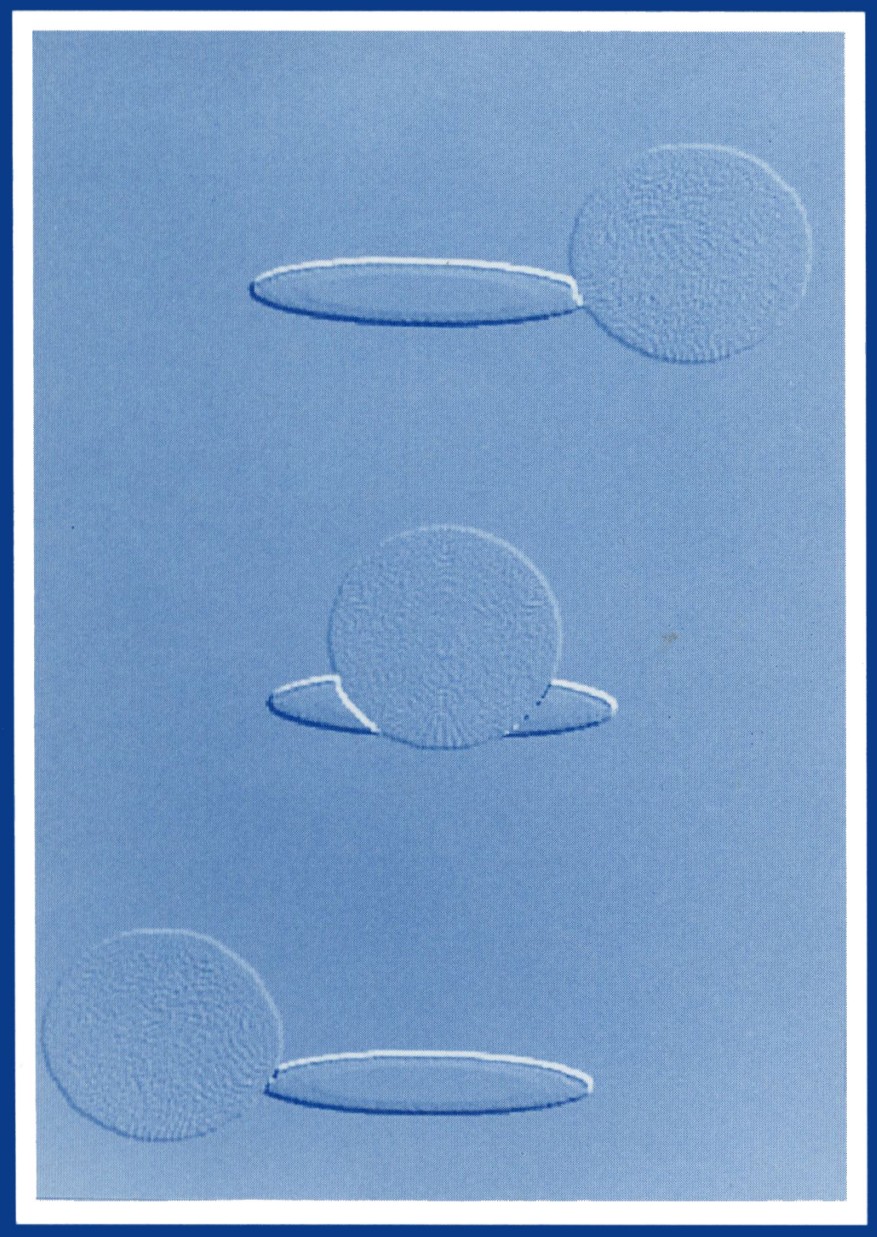

Kluwer Academic Publishers 
CATACLYSMIC VARIABLES AND RELATED OBJECTS 


\section{ASTROPHYSICS AND SPACE SCIENCE LIBRARY}

VOLUME 208

\section{Executive Committee}

W. B. BURTON, Sterrewacht, Leiden, The Netherlands

J. M. E. KUIJPERS, Faculty of Science, Nijmegen, The Netherlands

E. P. J. VAN DEN HEUVEL, Astronomical Institute, University of Amsterdam, The Netherlands

H. VAN DER LAAN, Astronomical Institute, University of Utrecht, The Netherlands

\section{Editorial Board}

I. APPENZELLER, Landessternwarte Heidelberg-Königstuhl, Germany

J. N. BAHCALL, The Institute for Advanced Study, Princeton, U.S.A.

F. BERTOLA, Università di Padova, Italy

W. B. BURTON, Sterrewacht, Leiden, The Netherlands

J. P. CASSINELLI, University of Wisconsin, Madison, U.S.A.

C. J. CESARSKY, Centre d'Etudes de Saclay, Gif-sur-Yvette Cedex, France

J. M. E. KUIJPERS, Faculty of Science, Nijmegen, The Netherlands

R. McCRAY, University of Colorado, JILA, Boulder, U.S.A.

P. G. MURDIN, Royal Greenwich Observatory, Cambridge, U.K.

F. PACINI, Istituto Astronomia Arcetri, Firenze, Italy

V. RADHAKRISHNAN, Raman Research Institute, Bangalore, India

F. H. SHU, University of California, Berkeley, U.S.A.

B. V. SOMOV, Astronomical Institute, Moscow State University, Russia

R. A. SUNYAEV, Space Research Institute, Moscow, Russia

S. TREMAINE, CITA, University of Toronto, Canada

Y. TANAKA, Institute of Space \& Astronautical Science, Kanagawa, Japan

E. P. J. VAN DEN HEUVEL, Astronomical Institute, University of Amsterdam, The Netherlands

H. VAN DER LAAN, Astronomical Institute, University of Utrecht, The Netherlands

N. O. WEISS, University of Cambridge, U.K. 


\title{
CATACLYSMIC VARIABLES AND RELATED OBJECTS
}

PROCEEDINGS OF THE 158TH COLLOQUIUM OF THE INTERNATIONAL ASTRONOMICAL UNION, HELD AT KEELE, UNITED KINGDOM, JUNE 26-30, 1995

\author{
Edited by \\ A. EVANS \\ and \\ JANET H. WOOD \\ Keele University, United Kingdom
}

KLUWER ACADEMIC PUBLISHERS DORDRECHT / BOSTON / LONDON 
A C.I.P. Catalogue record for this book is available from the Library of Congress.

Published by Kluwer Academic Publishers, P.O. Box 17, 3300 AA Dordrecht, The Netherlands.

Kluwer Academic Publishers incorporates the publishing programmes of

D. Reidel, Martinus Nijhoff, Dr W. Junk and MTP Press.

Sold and distributed in the U.S.A. and Canada by Kluwer Academic Publishers,

101 Philip Drive, Norwell, MA 02061, U.S.A.

In all other countries, sold and distributed

by Kluwer Academic Publishers Group,

P.O. Box 322, 3300 AH Dordrecht, The Netherlands.

Printed on acid-free paper

All Rights Reserved

(C) 1996 Kluwer Academic Publishers

No part of the material protected by this copyright notice may be reproduced or utilized in any form or by any means, electronic or mechanical, including photocopying, recording or by any information storage and retrieval system, without written permission from the copyright owner.

Printed in the Netherlands 


\section{CONTENTS}

Section headings and reviews are listed in boldface type

Preface:

List of participants:

NON-MAGNETIC CATACLYSMIC VARIABLES $\ldots \ldots \ldots \ldots \ldots \ldots \ldots \ldots 1$

The Nova-like variables: ....................................... V.S. Dhillon

The nova-likes AC Cnc, RW Tri, UX UMa and the nova DQ Her: common and different properties: ................................................. 13

E. Dmitrienko

Temperature structure of the disk in V1315 Aqr:....................... 15

C. Moreno, R.G.M. Rutten, V.S. Dhillon

Spectrally resolved maps of optically thick accretion disks: .................. 17

R. Baptista, K. Horne, I. Hubeny et al.

Roche tomography of the cool star in IP Peg:........................... 21

R.G.M. Rutten, V.S. Dhillon

Applications of indirect accretion disc imaging to orbital solutions for primary stars: 25 Martin Still

Distances and absolute magnitudes of a sample of faint cataclysmic variables:

L.N. Sproats, S.B. Howell, K.O. Mason

Studies in flickering:

W.F. Welsh, J.H. Wood, K. Horne

Decomposing interacting binary light curves: the eclipses of the mean light, secular variability and flickering in RW Tri:

Paul Bennie, R.W. Hilditch, K. Horne

The place of origin of the flickering in $\mathrm{Z}$ Cha:

Albert Bruch

TT Ari-94: A study of 1.6-60 minute variability:

I.L. Andronov, K. Arai, L.L. Chinarova et al.

Time resolved optical spectroscopy of V795 Her:

R.J. Dickinson, R.K. Prinja, S.R. Rosen, K. Horne

HST UV observations of the disk and wind of V795 Her:

S.R. Rosen, R.K. Prinja, J.E. Drew et al.

Dwarf nova outbursts and superoutbursts: .

J. Smak

IUE and optical spectra of RZ LMi and ER UMa throughout their 19 and 43 day

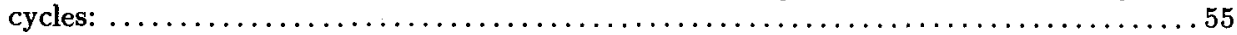

P. Szkody, A. Silber, R.K. Honeycutt et al.

New observational frontiers of ER UMa-type dwarf novae:.................... 59

D. Nogami, T. Kato, S. Masuda et al. 
Superhumps in the SU UMa star AK Cnc:..............................61

R. Mennickent, D. Nogami, T. Kato, W. Worraker

Outburst activity data on selected cataclysmic variables: .

T. Vanmuster, S.B. Howell

Eclipse observations of IP Peg during outburst:

W.J. Worraker, T. Naylor, G. Poyner, S.J. Robinson

Slingshot prominences during dwarf nova outbursts:.......................71 D. Steeghs, K. Horne, T.R. Marsh

Results of optical monitoring of the dwarf nova SS Cyg during the 1993 campaign of co-ordinated observations with the ASCA satellite: $\ldots \ldots \ldots \ldots \ldots \ldots \ldots \ldots \ldots \ldots \ldots$

I. Voloshina

The long period dwarf nova UY Pup in outburst: ........................ 75

J.J. Lockley, J.H. Wood, D.H.P. Jones, S. Mineshige

Observation of the WZ Sge-type dwarf nova AL Com:.

T. Kato, D. Nogami, H. Baba et al.

Simultaneous multiwavelength observations of dwarf novae systems at quiescence and in outburst: ................................................... 79

G. Tovmassian, J. Echevarria, E. Perez et al.

Is there observational evidence for the evaporation of the inner accretion disk in dwarf

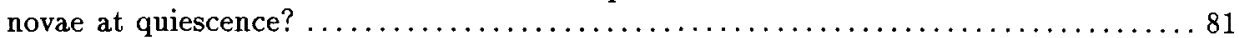

C. la Dous, E. Meyer-Hofmeister, F. Meyer

The temperatures of white dwarfs in dwarf novae:....................... 85

B.J.M. Hassall, C. la Dous

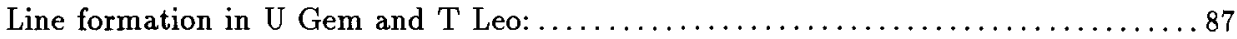

W. Hummel, K. Horne, T.R. Marsh, J.H. Wood

Population studies of cataclysmic variables: .......................... 89

F.A. Ringwald

Spectrophotometry of 106 to-be-confirmed CVs:

T. Zwitter, U. Munari, A. Bragaglia

ACCRETION DISCS

Accretion disc viscosity:

C. Tout

Dynamo driven accretion discs and dwarf nova eruptions:

P.J. Armitage, M. Livio, J.E. Pringle

On the turbulent viscosity prescription in accretion discs: $\ldots \ldots \ldots \ldots \ldots \ldots \ldots \ldots . \ldots 109$

P. Godon

The rise and fall of the $\alpha$-model viscosity:

F.V. Hessman, C. Obach

SPH simulations of tidally unstable accretion disks:

J. Murray

Inner edge drag by an asynchronous primary and accretion disc structure in close

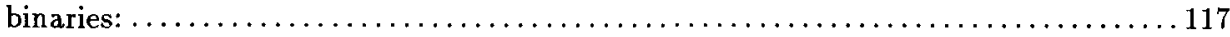

G. Lanzafame, G. Belvedere, D. Molteni

Synthetic spectra for accretion discs: the UV absorption spectrum:

R.A. Wade

A quantitative study of limb darkening in accretion disks:

M.P. Diaz, R.A. Wade, I. Hubeny 
Physical parameter mapping of accretion disks:

S. Vrielmann, F.V. Hessman, K. Horne, R. Baptista

Thermal-tidal instability model of dwarf novae below the period gap: a unification

theory:

Y. Osaki

Non circular disks in AM CVn systems?

J.-E. Solheim

Model spectra for the helinm cataclysmic variable: AM CVn:

S. Bard

Disk instabilities in the black hole binaries:

$J . K$. Cannizzo

The accretion disk model for the first hundred days of the outburst evolution in the black hole X-ray novae: ............................................. 139 S.-W. Kim, J.C. Wheeler, S. Mineshige

INTERMEDIATE POLARS

The intermediate polars:

C. Hellier

Mass and angular momentum flows in magnetic CVs:

A.R. King

Accretion disc formation in intermediate polars:

G.A. Wynn, A.R. King

The flares of AE Aqr:

M. Eracleous, K. Horne, M. Livio, W. Welsh

Triggering the radio emission from AE Aqr: ............................ 167

D. Steeghs, J. Kuijpers, L. Fletcher et al.

HST FOS observations of YY Dra:

C.A. Haswell, J. Patterson

The 71-second oscillation of DQ Her at $2180 \AA$ :

E.L. Robinson, E. Zhang, R.C. Bless et al.

Spin-resolved $\mathrm{H} \alpha$ spectroscopy and photometry of the intermediate polar

RX J0558+5353:

S.R. Duck, M.D. Still, A. Allan et al.

Rotational disturbance in $\mathrm{BG} \mathrm{CMi}$ :

M.A. Garlick

Simultaneous $U B V R I J K$ photometric and polarimetric observations of PQ Gem: ... 181 S. Potter, K.O. Mason, M.S. Cropper et al.

The magnetic field of the intermediate polar RE $0751+14$ :

H. Väth

On the long-term light curve behaviour of the intermediate polar TX Col:

D.A.H. Buckley

POLAR SYSTEMS

Magnetic fields and accretion streams in polars:

A.D. Schwope

Radiation hydrodynamics of accreting magnetic white dwarfs:

K. Beuermann, U. Woelk 
Magnetic fields in AM Her binaries:

K. Wu, P.A. Mason

The hard X-ray spectra of EF Eri and other CVs:

C. Done, J.P. Osborne, A.P. Beardmore

Different types of photometric and polarimetric behaviour of AM Her in

1989-1994:

N.M. Shakhovskoy, I.L. Andronov, S.V. Kolesnikov

Soft X-ray flickering of AM Her:.

P.J. Wheatley

X-ray and optical observations of BY Cam

G. Ramsay, P.A. Mason

Asynchronous rotation in BY Cam: it's got a good beat and you can dance to it (very slowly):

A.D. Silber

QPOs of BY Cam in low and high states:

E.P Pavlenko, S. Yu. Shugarov, S. V. Antipin et al.

Long-term brightness changes of two CVs: ...

D.A. Sokolov, S. Yu. Shugarov, E.P. Pavlenko

Optical flickering and shot noise in AM Her systems:

$S$. Larsson, B. Larsson

High speed UV photometry of the AM Her systems AM Her, VV Pup, and V834 Cen with the Hubble Space Telescope: ................................... 223

K. Schaefer, H. Bond, G. Chanmugam

Recent progress; in the polar QS Tel: the HST results:

K.L. Clayton, S.R. Rosen, J.P. Osborne

QQ Vul revisited:

M.S. Catalán, S. Davey, R. Smith, D. Jones

RX J1015.5+0904: a new polar at the lower period limit:

V. Burwitz, K. Reinsch, A.D. Schwope et al.

SPACE OBSERVATIONS

Far ultraviolet observations of dwarf novae made with the Hopkins Ultraviolet Telescope:

Knox S. Long

EUVE observations of VW Hyi in superoutburst:

C.W. Mauche

The possible contribution from the accretion disk during the quiescence of

VW Hyi:

M. Huang, E.M. Sion, I. Hubeny et al.

Preliminary analysis of a Hubble FOS spectrum of VW Hyi in quiescence: a DAZQ white dwarf and accretion belt/ring: .......................................249 E.M. Sion, M. Huang, F.H. Cheng et al.

VW Hyi: a rapidly cooling white dwarf?

B.T. Gänsicke, K. Beuermann

Accretion disk winds in cataclysmic variables:

C. Knigge, J.E. Drew, K.O. Mason

The transient EUV sky as observed by ALEXIS:

D. Roussel-Dupré, J.J. Bloch 
ASCA observations of cataclysmic variables:

M. Ishida, R. Fujimoto, K. Matsuzaki

An ASCA observation of the eclipsing dwarf novae HT Cas:

K. Mukai, E.M. Schlegel, J.H. Swank et al.

ROSAT observations of non-magnetic CVs:

$A$. van Teeseling, $F$. Verbunt, $K$. Beuermann

COMPTEL observations of X-ray binaries:

A.F. Iyudin

NOVAE AND SYMBIOTIC STARS

Classical nova evolution: clues from soft X-ray emission:

J. MacDonald

The ROSAT observations of classical novae:

M. Orio, H. Ögelman, S. Balman

Observations of novae in M51, M87 and M101: a preliminary report: .

A.W. Shafter, R. Ciardullo, C.J. Pritchet

Are novae standard candles?

S. Pistinner, G. Shaviv, P.H. Hauschildt, S. Starrfield

TNR on top of accreting WDs: 2-D simulations:

S.A. Glasner, E. Livne, J.W. Truran

Nova ejecta abundances resulting from multi-cycle evolutionary calculations:

D. Prialnik, A. Kovetz

The gamma-ray spectrum of classical novae:

M. Hernanz, J. José, J. Gómez

The nature of GQ Mus as depicted from photoionization models of the shell: ...... 305

D. Péquignot, C. Morisset

Nova outbursts on rotating oblate white dwarfs:

A.D. Scott

Optical imaging of old nova shells:

T.J. O'Brien, A.J. Slavin

Shaping of nova shells by binary motion:

H.M. Lloyd, T.J. O'Brien, M.F. Bode

Ultraviolet spectropolarimetry of classical novae in outburst: evidence for aspherical

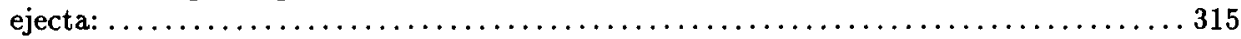

J.J. Johnson, C.M. Anderson, K.S. Bjorkman et al.

A unified model for the radio and optical images and spectra of nova

V1974 Cyg 1992:............................................... 317

R.M.Hjellming

V1974 Cyg (Nova Cyg 1992) - a possible link to the SU UMa stars:

A. Retter, E.M. Leibowitz, E.O. Ofek

$U B V$ photometry of V705 Cas (Nova Cas 1993) during the first year of outburst:...323 K. Petrik, L. Hric, Z. Urban et al.

Infrared spectroscopy of Nova V705 Cas:

A. Evans, T.R. Geballe, J.M.C. Rawlings, A.D. Scott

Detection of the irradiated red dwarf in WY Sge (Nova 1783):

M.W. Somers, K. Mukai, T. Naylor, F.A. Ringwald

The spectrum of the old nova V603 Aql observed with the HST:

M. Friedjung, P.L. Selvelli, A. Cassatella 
A new type of asymmetric outflow in the recurrent nova RS Oph in quiescence: ... 331 T. Iijima

V1016 Cyg: proper motion of radio emission:

S.P.S. Eyres, S.M. Dougherty, R.J. Davis et al.

Evolution of an accretion disk in the symbiotic binary CI Cyg:

J. Mikolajewska

The peculiar symbiotic star CH Cyg - a bright counterpart of cataclysmic variables?

\section{Leedjärv}

Possible periodic components in the flickering of $\mathrm{CH}$ Cyg and MWC 560:

M. Mikolajewski, T. Tomov, A. Dapergolas, Y. Bellas-Velidis

A close look at the outburst of the very slow symbiotic nova PU Vul - a triple

system:

D. Chochol, S. Tamura, O. Kanamitsu et al.

A unique symbiotic-like/cataclysmic triple star 4 Dra: six years of $U B V$

monitoring:.

Z. Urban, L. Hric, L. Leedjärv

X-RAY BINARIES

Observations of low mass X-ray binaries:

A.P. Smale

Generation of X-ray fluctuations in X-ray binaries:

S. Mineshige, M. Takeuchi, H. Negoro

Cyg X-2: its recurrent X-ray behaviour:

E. Kuulkers, M. van der Klis

The orbital period of the Galactic $\mathrm{Z}$ source Sco X-2:

K.A. Southwell, J. Casares, P.A. Charles

Optical counterpart of the LMXB GX 13+1:

P.J. Groot, M. van der Klis, J. van Paradijs et al.

Detection of the secondary star in X1822-371:

E.T. Harlaftis, P.A. Charles, K. Horne

Radio emitting X-ray binary stars:

R.E. Spencer

MERLIN observations of extended emission around GRS 1915+105:

S.J. Newell, R.E. Spencer, F.H. Jowett

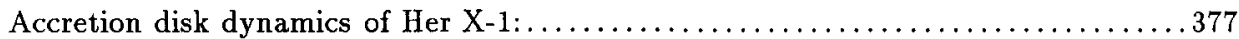

S.D. Vrtilek, F.H. Cheng

An archival study of HST observations of Her X-1/HZ Her:

F.H. Cheng, S.D. Vrtilek, J.C. Raymond

The 35 day cycle of Her X-1:

S. Schandl, F. Meyer

Models of soft X-ray transients and dwarf novae:

J.P Lasota

Doppler imaging of V404 Cyg in quiescence:

J. Casares

The X-ray nova GRO J0422+32 in decline and quiescence:

M.R. Garcia, P.J. Callanan, J.E. McClintock, P. Zhao 
Investigation of the optical variability of GRO J0422+32:

M. Popova, G. Beskin, S. Mitronova et al.

Investigation of the optical variability of relativistic objects with high time

resolution:

S.N. Mitronova, G.M. Beskin, S.I. Neizvestny et al.

Accreting white dwarfs and Type Ia supernovae:

M. Livio, D. Branch, L.R. Yungelson et al.

A model for the Galactic population of supersoft X-ray sources:

L. Yungelson, A. Tutukov, A. Fedorova et al.

The period distribution of supersoft sources:

P. Kahabka

ROSAT and optical observations of supersoft X-ray sources:

$K$. Reinsch, A. van Teeseling, K. Beuermann et al.

Visual light from the eclipsing supersoft X-ray source CAL 87:

S. Schandl, E. Meyer-Hofmeister, F. Meyer

The supersoft source RX J0019.8+2156: new photoelectric observations:. . . . . . . 427

C. Bartolini, A. Guarnieri, A. Piccioni, L. Solmi

The supersoft X-ray source RX J0537.6-7033:

M. Orio, M. Della Valle, G. Massone, H. Ögelman

POST COMMON ENVELOPE BINARIES AND EVOLUTION

Understanding the long-term evolution of CVs and LMXBs: principles and problems: ...................................................433 U. Kolb

Simulations of angular momentum evolution in wind-fed CV precursors: .........443 K.J. Pearson, G.A. Wynn, A.R. King

A core-envelope decoupling dynamo model for cataclysmic variables:

L. Zangrilli, C.A. Tout, A. Bianchini

Nova outbursts and the secular evolution of cataclysmic variables:

K. Schenker, U. Kolb, H. Ritter

Secular evolution of cataclysmic variables with irradiation-induced mass transfer: .. 449 H. Ritter, Z. Zhang, J.M. Hameury

From common envelope to pre-cataclysmic variables: an observational test of common envelope evolution:

M.J. Sarna

The ${ }^{12} \mathrm{C} /{ }^{13} \mathrm{C}$ ratio as a tracer of the evolution of post common envelope systems and cataclysmic variables: ......................................... 457 M.J. Sarna, P.B. Marks, R.C. Smith

Evolutionary scenarios for double degenerate systems:

P.B. Marks, M.J. Sarna, R.C. Smith

On the origin of the abundance anomalies in IK Peg:

B. Smalley, K.C. Smith, D. Wonnacott

The extended dust emission around GK Per:

S.M. Dougherty, L.B.F.M. Waters, M.F. Bode et al. 
Low mass white dwarfs in binaries: . ............................... 465

T.R. Marsh, V.S. Dhillon, S.R. Duck

Hot subdwarfs in binaries: multiwavelength observations and evolutionary in plications: ................................................ 469 A. Ulla, P. Thejll, S. Vennes et al.

The eclipses of the close binary star BE UMa:

J.H. Wood, E.L. Robinson, E.-H. Zhang

An evolutionary scenario for short period ( $\leq 10$ days) millisecond binary pulsars: .. 473 E. Ergma, M.J. Sarna, J. Antipova

On the orbital period distribution of recycled pulsars: 475 L.A. Nelson, S. Rappaport

CONFERENCE SUMMARY

Keele cataclysmic commentary:

B. Warner

Subject index

Object index 